\title{
Depósitos eólicos del trópico húmedo: caso de la franja marino-costera del este de la Península de Azuero, Panamá
}

\author{
Aeolian deposits of the humid tropics: case of the marine- \\ coastal strip of the eastern part of the Azuero Peninsula, \\ Panama
}

\author{
Jaime A. Rivera-Solís ${ }^{1}$ \\ Universidad de Panamá, Panamá
}

\begin{abstract}
Resumen
La pesquisa tuvo por objeto demostrar la incidencia del viento como agente morfogenético, a través de la correlación de los clastos de arenas que constituyen las geofacies marino costeras. Se seleccionó la teoría que discute los limites morfoclimáticos y se compilan los datos de temperatura, precipitación y vientos; en campo, se clasifican las geoformas de acumulación, erosión y se realiza el muestreo de arenas por geofacies. Las muestras de arena (453 g), son tamizadas para separar sus componentes y obtener el tamaño de los clastos, seguidamente, expuestas al estereomicroscopio para determinar redondez y esfericidad por comparación visual. Posteriormente, la muestra de la planicie costera $(1 \mathrm{~g})$, se trata $1 \mathrm{~N}(\mathrm{~K} 2 \mathrm{Cr} 2 \mathrm{O} 7)$ y $(\mathrm{H} 2 \mathrm{SO} 4)$, para la reducción de materia orgánica y determinar su génesis. Finalmente, utilizando las arenas del mesolitoral como variable independiente para evidenciar la correlación entre éstas y el origen de los depósitos eólicos por geofacie, se comprueba que, con las dunas litorales existe una correlación lineal positiva y coeficiente perfecto $(\rho \pm 0,98)$, con las dunas nebkha una correlación lineal positiva de coeficiente perfecto $(\rho \pm 0,98)$, y con respecto al manto de arenas una correlación lineal positiva de coeficiente perfecto $(\rho \pm 0,96)$.
\end{abstract}

Palabras clave: Erosión eólica, clastos de arenas, depósitos eólicos, clima tropical.

\section{Abstract}

The purpose of the investigation was to demonstrate the incidence of wind as a morphogenetic agent through the correlation of the sand clasts that constitute the marine-coastal geofacies. Morphoclimatic limit theory was selected and the data on temperature, precipitation and winds compiled;

1 Doctor en Geografía. Universidad de Panamá. Profesor Titular del Departamento de Geografía Física. CCIMBIO: Centro de Capacitación, Investigación y Monitoreo de la Biodiversidad, CRUV-UP. Correo jaime.rivera@up.ac.pa (D) https://orcid.org/0000-0002-8265-7836 
field activities included the classification of the geoforms of accumulation and erosion as well as the sampling of sands per geofacies. Sand samples ( $453 \mathrm{~g}$ ) were sieved to separate their components and assay clast sizes, followed by stereomicroscopic examination to determine roundness and sphericity by visual comparison. Subsequently, the sample from the coastal plain $(1 \mathrm{~g})$ was treated with $1 \mathrm{~N}$ potassium dichromate solution $\left(\mathrm{K}_{2} \mathrm{Cr}_{2} \mathrm{O}_{7}\right)$ and sulfuric acid $\left(\mathrm{H}_{2} \mathrm{SO}_{4}\right)$, to oxidize and eliminate organic matter and determine its genesis. Finally, the correlation between mesolittoral sand as independent variable and origin of aeolian deposits per geofacies was found to be of positive linear nature with high correlation coefficients $(\rho)$ for coastal dunes $(\rho \pm 0.98)$, nebka dunes $(\rho \pm 0.98)$ and sand mantles $(\rho \pm 0.96)$.

Keywords: Wind erosion; Sand clasts; Aeolian deposits; Tropical climate.

\section{Introducción}

Los resultados morfogenéticos del viento son un factor geológico cuyos efectos se relacionan habitualmente con los climas áridos y semiáridos, sin embargo, es necesario documentar su capacidad morfodinámica en los climas tropicales húmedos y subhúmedos, como responsables por la elaboración y el modelado de los ambientes residuales (geoformas de erosión), y de deposición (geoformas de acumulación), que integran el paisaje natural.

La pesquisa no pretende abordar el espacio natural (marino costero), a través de teorías científicas puras de Geomorfología Dinámica y/o Climática; no obstante, para defender profesionalmente la propuesta de ordenación ambiental, Sánchez (2003, p. 17), considera que el objetivo de los diagnósticos ambientales es comprender los mecanismos de funcionamiento de los diversos ambientes, siendo necesario investigar cada uno de los elementos de ese espacio, lo cual también incluye la comprensión de la distribución del relieve sobre la superficie, puntualizando forma, dinámica y origen.

Es así, a través de esta visión de sustentabilidad ambiental del paisaje, donde la Geomorfología Ambiental justifica su campo de estudio procurando, según Teixeira y Dos Santos (2015, pp. 17 - 18), ofrecer diagnósticos y soluciones a los conflictos ambientales que surgen en la sociedad, analizando sistémicamente el espacio natural y las actividades antrópicas desarrolladas.

Dentro de éste contexto, surge la investigación a raíz del estudio del espacio natural que realiza el geógrafo en Panamá, amparados en la ley 6 de ordenamiento territorial y la resolución No. 4 de 2009, que contemplan los requisitos y regulan los contenidos técnicos que aplican 
al solicitar una asignación de uso de suelo, o al presentar un plan de ordenamiento territorial.

Así, las investigaciones para la ordenación del espacio se vislumbran como un instrumento de gestión ambiental preventivo indirecto que contienen el detalle de las unidades geomorfológicas que integran el geosistema; mientras que, como estrategia de planificación los planes de ordenamiento territorial, son un instrumento de gestión ambiental preventivo directo que cumplen la función de recomendar y regular el uso del espacio a través de leyes procurando garantizar su sustentabilidad.

Al respecto, para Díaz (2011, p. 102), la sustentabilidad del paisaje natural incluye el análisis de la dimensión geomorfológica, puesto que, ésta variable ofrece la medida física que permite dilucidar las geoformas que adopta el paisaje producto de los flujos de energía; los cuales, son necesarios comprender para evitar el colapso del espacio y garantizar su resilencia ambiental.

A saber, latitudinalmente, la República de Panamá se ubica al norte del ecuador geográfico y se extiende $270,43 \mathrm{~km}$ desde el punto cardinal norte con $9^{\circ} 38^{\prime} 46.2^{\prime} \mathrm{N}$ (Isla Tambor), hasta el punto cardinal sur (Isla Jicarita), con $7^{\circ} 12^{\prime} 07.8^{\prime} \mathrm{N}$ (Atlas, 2016); efectivamente, pertenece a la zona húmeda ecuatorial, la cual registra las mayores precipitaciones pluviales del planeta con valores anuales $>1000 \mathrm{~mm}$ (Craig; Vaughan y Skinner, 2012, p. 424).

Cabe agregar que, el límite inferior de la isoyeta requerida para la evolución de los paisajes de la zona subhúmeda es $>1000 \mathrm{~mm}$ (Mateo, 2012, p. 83), y para la zona húmeda $>1400$ mm (Gutiérrez, 2009, p. 173; Mateo, 2012, p. 83); y respectivamente, pueden presentar en promedio de tres a cinco meses secos (Troppmair, 2012, p. 54).

Al respecto, diversos investigadores han realizado aportes abordando el tema de la delimitación de los dominios climáticos utilizando el análisis comparado de los parámetros de temperaturas, precipitaciones y periodos secos, como variables meteorológicas para facilitar la regionalización climática.

Sin embargo, merece destacar los aportes de la escuela alemana de V. Köppen (1846-1940), quien elaboró su reconocida clasificación climática en 1900, modificada entre 1918-1923, y posteriormente suscrita en compañía de R. Geiger en 1936 (Peel, Finlayson y McMahon, 2007, p. 
1635), la cual por su afable interpretación y rigor científico continúa siendo aplicada por un sinnúmero de geógrafos y meteorólogos.

Desde sus inicios la citada clasificación se utiliza combinando letras que indican las características de temperatura, precipitación y la escasez de agua. Así, en la región ecuatorial (Que se extiende hasta los $10^{\circ}$ de latitud norte y sur), se encuentra el clima tropical lluvioso identificado con la letra "A" para discriminar el comportamiento de la temperatura cuyos valores deben ser $>18^{\circ} \mathrm{C}$, y para destacar los efectos de la estacionalidad climática a través de la existencia o no de un periodo seco, aplica letras minúsculas donde para el caso, la letra "f" (Fehlt), indica ausencia de periodo seco, y "w" (Winter), para destacar que existe periodo seco durante el invierno.

Cabe acotar que el clima tropical (A), como sistema, acusa los efectos meteorológicos que genera el desplazamiento de la zona de convergencia intertropical: Periodos lluviosos y periodos secos.

Este fenómeno se refleja en la zona de pesquisa, producto del movimiento de la zona de convergencia intertropical que, al trasladarse hacia el sur, durante el verano del hemisferio austral, se ubica casi encima del ecuador geográfico (Ayoade, 2011, p. 125); y, en consecuencia, puesto que la cobertura de nubes se retira, los vientos alisios del NE y ENE invaden el pacífico panameño provocando el inicio el periodo seco (Enero - abril).

Éste periodo se caracteriza por la presencia de constantes ráfagas de aire fresco y seco que "descienden y se calientan por la proximidad de la superficie terrestre, aumentando rápidamente su capacidad de contener el agua" (Craig et al., 2012, p.425); ocasionando estragos en el sector agropecuario por stress hídrico, además de los efectos de la erosión causada por los vientos que degradan las tierras agrícolas al remover la capa superficial de suelo durante la estación seca en las regiones subhúmedas (Ayoade, 2011, p. 269), húmedas, o caso contrario por el revestimiento del suelo con sedimentos transportados, los cuales podrían provocar su acidificación y elevar los costos de producción anual asociados a la inversión de agroquímicos y fertilizantes para preparar el suelo.

Por ende, la existencia del periodo seco acusa la necesidad de discutir sus efectos morfogenéticos, ponderando la ocurrencia de la estacionalidad climática por medio del registro de la temperatura (Mes más cálido) 
y el total de meses con precipitación > $50 \mathrm{~mm}$ (Gutiérrez, 2009, p. 445), incluyendo ahora la dirección y velocidad del viento como agente morfogenético responsable por la creación de ambientes marino costeros de erosión y acreción.

\section{Área de estudio}

Ya citado, la investigación se lleva a cabo en la República de Panamá. El área de estudio involucra del pacífico panameño, el litoral E de la península de Azuero, del cual, el polígono de muestreo totaliza 96.2 ha de la planicie costera. Los puntos costeros que delimitan la finca se encuentran entre las coordenadas UTM - 17N/ 843046,00 / N y 604099,00 / E (P4), con 844135,00 / N y 603732,00 / E (P3); y la franja frente al litoral se extiende 1,54 km entre las coordenadas UTM - 17/ N/ 844499,00/ N y 604686,00 / E (P1), con UTM - 17/ N/ 843363,00 / N y 604862,00 / E (P2), (Figura 1).

$\mathrm{Al}$ respecto, sobre el área de estudio, un antecedente que demuestra la posibilidad de que, sobre la planicie costera se genere un ambiente de depositación, queda expuesto cuando a través de un corte calicata (UTM $17 \mathrm{~N} / 843743,00 / \mathrm{N}$ y $604362,00 / \mathrm{E}$ ), realizado en el mes de julio (periodo lluvioso), Rivera (2016), indica que el primer horizonte del suelo con profundidad de 0 a $16 \mathrm{~cm}$ presenta un $\mathrm{pH}$ de 5,6 (ácido moderado), con $32 \%$ de arena, $48 \%$ de limo y $20 \%$ de arcilla; datos los cuales, interpretados en el diagrama triangular de clasificación textural de suelos, determinan una capa franco limoso.

En consecuencia, los citados resultados justifican la necesidad de realizar una nueva calicata durante el periodo seco, para comprobar la concentración de las partículas del primer horizonte y su pH. El segundo muestreo de suelos (UTM - 17N/ 843585,00/ N y 604157,00/ E), se realizó en el mes de abril de 2019 (periodo seco), resultando que el primer horizonte con profundidad de 0 a $27 \mathrm{~cm}$ presenta un $\mathrm{pH}$ de 4,2 (Extremadamente ácido), con $84 \%$ de arena, $8 \%$ de limo y $8 \%$ de arcilla; donde, el análisis textural revela la conformación de un horizonte de arena poco franca. 
Figura 1. Ubicación regional del punto de pesquisa (Superior), y polígono ampliado (Inferior). (Rivera, 2020).

Figure 1. Regional location of the research point (Upper), and expanded polygon (Lower). (Rivera, 2020).

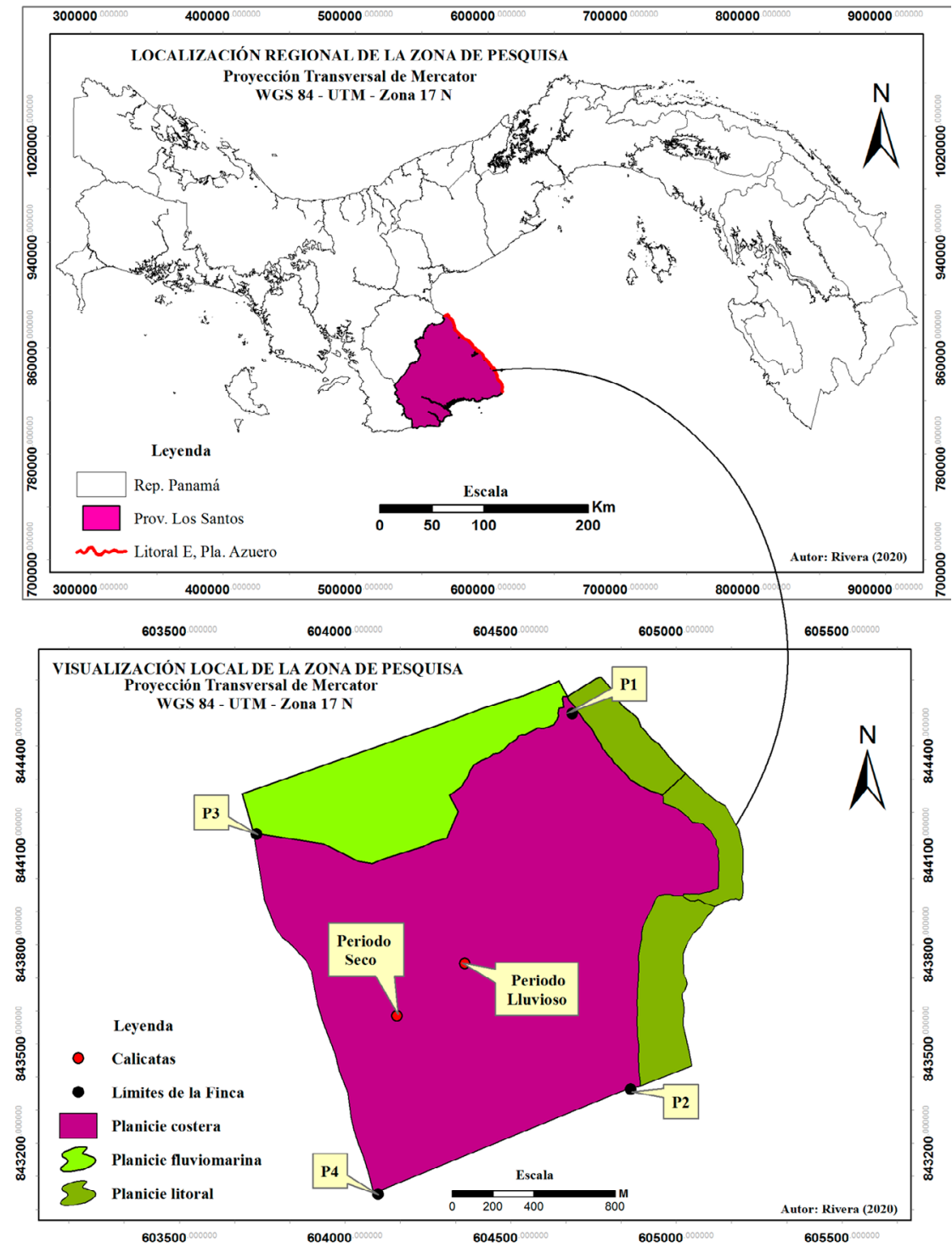


La desertificación es un proceso que ocasiona la degradación ambiental del geosistema, y genera consecuencias socioambientales por la pérdida de su capacidad productiva; sus efectos, son desencadenados por la escases de agua y la erosión eólica, los cuales propician la acidificación del suelo (Craig et al., 2012, p. 472; Mateo, 2012, p. 90).

Así, producto de la variante textural y la disminución del $\mathrm{pH}$ que presentan las muestras comparadas del primer horizonte, la pesquisa tuvo por objeto demostrar la incidencia del viento como agente morfogenético al correlacionar los tipos de arenas que constituyen las geofaces marino costeras determinando las propiedades morfológicas del clasto y su génesis; puesto que, por clasificación textural, la arena se define por tamaño del clasto, y no por su fuente de origen.

\section{Marco metodológico}

Con base en el alcance de los procedimientos aplicados, la investigación se proyecta observacional cuantitativa de tipo analítico; porque, procura comprobar o desestimar la relación entre variables por correlación, utilizando un grupo de control. De secuencia transversal al abordar un grupo específico de variables que estadísticamente permiten demostrar asociaciones positivas o negativas en un momento determinado (año 2019), y de carácter retrospectivo, puesto que, la colecta de datos (climáticos), parte de observaciones pretéritas.

Con base en los resultados esperados, sustenta el movimiento de las de arenas, al determinar una relación entre el tamaño del clasto y la velocidad del viento; expresando los resultados por medio de variables estadísticas cuantitativas continuas, además de caracterizar las geoformas expuestas.

\section{Materiales}

- Datos climáticos: Estación Pedasí (dato histórico de precipitación y temperatura. Promedio mensual, anual y total. Registros de Años: 1967 - 2019), estación Los Santos (dato histórico viento. Promedio mensual, anual y total. Registros de Años: 1964 - 2019).

- Cámara digital fotográfica HD. Canon SX30-IS.

- Sistema de posicionamiento global: Garmin Etrex 20. GPS + GLONASS + WAAS.

- $\quad$ Clinómetro y cinta métrica.

- Un (1) kg. (2,20462 lb.), de muestra de suelo (Planicie costera). 
- Un (1) kg. (2,20462 lb.), de muestras de arenas por geoforma identificada.

- $\quad$ Sistema para geoprocesamiento: Software ArcGIS 10.

- Balanza digital.

- $\quad$ Muestra de arena de la planicie costera(1g).

- Dicromato de potasio 1N (K2Cr2O7), (10 cc).

- Ácido sulfúrico (H2SO4), (20cc).

- Ácido fosfórico ( $\left.\mathrm{H}_{3} \mathrm{PO} 4\right)(10 \mathrm{cc})$.

- $\quad$ Agua destilada (100cc).

- $\quad$ Sulfato de hierro pentahidratado (FeSO4.5H2O).

- $\quad$ Platos para tamizado: Mallas $\mathrm{N}^{\circ} 35(0,500 \mathrm{~mm}), 60(0,250 \mathrm{~mm}), 120$ $(0,125 \mathrm{~mm})$, y $230(0,063 \mathrm{~mm})$.

- $\quad$ Estereomicroscopio Leica LED3000 y placa de Petri.

- Tabla de comparación visual de redondez y esfericidad de arena, según Krumbein y Sloss (1963), citado por Kenitiro (1973, p. 113).

Procedimientos metodológicos

El estudio de la relación clima y formas resultantes ha sido abordada por reconocidos autores como Peltier y Chorlley, Strahler, Cailleux y Tricart, Wilson; entre otros hasta la actualidad. Éstas clasificaciones morfoclimáticas reflejan con propiedad la realidad ambiental del paisaje cuando la escala témporo-espacial se aplica a nivel de zona, dominio y región natural.

No obstante, para caracterizar las unidades del paisaje natural a nivel de geosistema, geofacie, y geotopo (Bertrand y Bertrand, 2007, p. 15), el estudio local debe incluir el efecto del viento como factor morfogenético en los climas estacionales del trópico subhúmedo, así como también en los tropicales húmedos los cuales registran precipitaciones $>1400 \mathrm{~mm}$.

1. Se analizan las teorías base sobre los principios de la Geomorfología Climática. Aceptando que, las geoformas derivan de un sistema de erosión determinado del clima, para definir la región morfogenética, el análisis del comportamiento de los datos de temperatura y precipitación, se realizará ponderando las clasificaciones de Peltier (1950), y Chorley et al. (1984), citados por Gutiérrez (2009, pp. 442 - 445); mientras que, se comprobará la relación entre la velocidad del viento y el diámetro del clasto transportado, según Blyth y De Freitas (1989, p. 79), Simons (1990, p. 501) y Gutiérrez (2009, pp. 356 - 358). 
2. Se compilan los datos climáticos de temperatura y precipitación de la estación Pedasí, ubicada a 47 msnm, más, los datos de viento que se obtienen de la estación Los Santos, ubicada a 16 msnm (Disponibles en: http://www.hidromet.com.pa/clima_historicos.php?sensor=1; http://www.hidromet.com.pa/clima_historicos.php?sensor=2 ; http://www.hidromet.com.pa/clima_historicos.php?sensor=6 ; y http://www.hidromet.com.pa/clima_historicos.php?sensor=7)

También, durante el periodo seco (2019), se verifican por 30 días los datos de velocidad y dirección del viento sobre el punto, con el auxilio del sitio Windfinder, cuya plataforma de consulta meteorológica utiliza una amplia red de estaciones meteorológicas $( \pm 21000)$, a nivel mundial, generando mapas de previsión eólica y datos sobre la velocidad y dirección del viento en tiempo real, en más de 45000 lugares de todo el mundo (Disponible en: https://es.windfinder. com/\#3/14.0936/-82.0054).

3. Para delimitar y cuantificar el espacio, en campo, a), se localizan los puntos perimetrales que definen el espacio de muestreo e integran el geosistema, b), se identifican las geofacies y se georeferencian sus polígonos, c), se colecta aproximadamente $1 \mathrm{~kg}$ de arena por geoforma y se georeferencian los puntos.

4. En el laboratorio: Se elabora el plano geomorfológico adoptando la simbología de Verstapen y Zuidam (1975, pp. 30 - 34), y atendiendo los requerimientos establecidos por las leyes de ordenamiento territorial (República de Panamá), las cuales ordenan el uso de escalas < 1:5 000.

5. Por muestreo aleatorio simple, se extraen $453 \mathrm{~g}$. de arenas a cada una de las muestras colectadas. Se realiza el análisis granulométrico, aplicando la técnica de diámetro del tamiz, se determina el tamaño de los clastos, utilizando las mallas $\mathrm{N}^{\mathrm{o}} 35,60,120$ y 230 .

6. Para comprobar si las partículas de arena que recubren la planicie costera son producto de la meteorización en In situ del suelo, o que, probablemente son transportados y depositados por el viento; por muestreo aleatorio simple se extrae $1 \mathrm{~g}$. de la muestra y se aplica el método de Walkley y Black (1934), citado por (Eyherabide, Saínz, Barbieri y Echeverría, 2014, p. 14) para la reducción de materia orgánica.

Dicho método consiste en la reducción de carbono (C), en fase húmeda de la muestra de suelo aplicando dicromato de potasio 
e incorporando ácido sulfúrico, lo cual genera la oxidación del C. La reacción reduce el dicromato (que equivale a la cantidad de $\mathrm{C}$ que se oxida), (Eyherabide, et al. 2014, p.14); posteriormente, el dicromato residual se titula con sulfato de hierro pentahidratado (FeSO4.5H2O) (por goteo), para determinar la concentración.

7. Para fijar los parámetros de redondez y esfericidad, la muestra depurada se coloca en la placa de petri y se remite al estereomicroscopio, procurando los clastos de cuarzo. Posteriormente, las imágenes obtenidas (De la planicie costera y el mesolitoral), se cotejan con la tabla de comparación visual de arena de Krumbein y Sloss (1963), citado por Kenitiro (1973, p. 113).

8. Para evidenciar la correspondencia morfogenética de los depósitos eólicos, se utiliza el coeficiente de correlación (r), como valor numérico para demostrar el grado de asociación entre la variable independiente y la dependiente, y cuyos límites de magnitud se sitúan entre -1 y 1 ; donde, para muchos autores, valores de $r=-1$ demuestran una correlación negativa grande y $\mathrm{r}=1$ demuestra una correlación positiva alta.

Al respecto, para Schober, Boer y Schwarte (2018, p. 1765), la correlación define el valor de una relación monotónica entre dos variables, las cuales pueden ser directamente o inversamente proporcionales. Además, el autor indica que, no existe unanimidad en la interpretación de los coeficientes de correlación intermedios, sin embargo, existe consenso entre los investigadores para definir las correlaciones de valores extremos; donde los coeficientes $<0,1$ indican una insignificante relación y los $>0,9$ una alta relación o muy fuerte relación. Cabe agregar que, éstos índices expresan el denominado coeficiente de correlación de Pearson: $r=x$.

\section{Resultados}

Por medio de los registros climáticos (Tabla 1), y las recomendaciones teorías citadas, se determina la región morfogenética (Figura 2), como tropical húmeda seca; puesto que, su temperatura media anual promedio $>$ $27^{\circ} \mathrm{C}$, su precipitación total anual promedio es de $1564,4 \mathrm{~mm}$, y la temperatura del mes más cálido de $28,1^{\circ} \mathrm{C}$.

Además, presenta ocho (8), meses con precipitación $>50 \mathrm{~mm}$ mensuales promedio (los resaltos en rojo sobre la figura 2 son nuestro). 
Respecto a los procesos geomorfológicos, se destaca que, con precipitaciones $>1520 \mathrm{~mm}$ anuales, se consideran los efectos morfodinámicos del viento como mínimos; mientras que, la erosión pluvial y los movimientos de masas son evaluados como moderados.

Para complementar, por medio de la plataforma de consulta meteorológica Windfinder, se verifico la dirección y velocidad del viento durante el mes de enero (2019), resultando una dirección constante de $\mathrm{N}$ y NE, con velocidad promedio $5 \mathrm{~m} / \mathrm{s}$.

Tabla 1. (Izquierda), temperatura y precipitación, según de la estación meteorológica Pedasí ( No 126-005 / 47 msnm). Años 1967 - 2019. (Derecha), velocidad máxima del viento, según datos de la estación meteorológica Los Santos ( $\left.\mathrm{N}^{\mathrm{o}} 128-001 / 16 \mathrm{msnm}\right)$.

Años 1964 - 2019. Autor. Rivera (2019).

Table 1. (Left), temperature and precipitation, according to the Pedasí weather station $\left(\mathrm{N}^{\circ} 126-005\right.$ / 47 masl). Years 1967 - 2019. (Right). Maximum wind speed, according to data from the Los Santos weather station $\left(\mathrm{N}^{\circ} 128-001 / 16\right.$ masl). Years 1964 - 2019.

Author. Rivera (2019).

\begin{tabular}{|c|c|c|}
\hline Mes & Temperatura $/{ }^{\circ} \mathrm{C}$ & Precipitación / mm \\
\hline Enero & 27 & 9.4 \\
\hline Febrero & 27.3 & 0.9 \\
\hline Marzo & 27.5 & 4.3 \\
\hline Abril & 28.1 & 23 \\
\hline Mayo & 27.8 & 39.6 \\
\hline Junio & 27.1 & 213.4 \\
\hline Julio & 27 & 246 \\
\hline Agosto & 26.9 & 249.5 \\
\hline Septiembre & 26.8 & 203.2 \\
\hline Octubre & 26.6 & 193.3 \\
\hline Noviembre & 26.6 & 200.9 \\
\hline Diciembre & 26.8 & \\
\hline Promedio / Total & 27.1 & 1564.4 \\
\hline
\end{tabular}

\begin{tabular}{|l|cc|}
\hline \multicolumn{2}{|c|}{ Mes } & \multicolumn{2}{c|}{ Viento $2 \mathrm{~m}(\mathrm{~m} / \mathrm{s})$} & Viento $10 \mathrm{~m}(\mathrm{~m} / \mathrm{s})$ \\
\hline Enero & 3.2 & 4.7 \\
\hline Febrero & 3.7 & 4.7 \\
\hline Marzo & 3.6 & 5.8 \\
\hline Abril & 2.9 & 4.7 \\
\hline Mayo & 2.2 & 3.6 \\
\hline Junio & 1 & 2.3 \\
\hline Julio & 1.4 & 2.2 \\
\hline Agosto & 1.2 & 2 \\
\hline Septiembre & 0.9 & 2.2 \\
\hline Octubre & 0.8 & 1.5 \\
\hline Noviembre & 1.5 & 2.4 \\
\hline Diciembre & 2.6 & 12.1 \\
\hline
\end{tabular}


Figura 2. (Izquierda), importancia de los procesos geomorlógicos en función de la temperatura media anual y la precipitación total anual, según Peltier (1950). (Derecha), regiones morfoclimáticas actuales, a partir de la temperatura media anual, precipitación total anual y número de meses húmedos por encima de $50 \mathrm{~mm}$., según Chorley et al. (1984); citados por Gutiérrez (2009, pp. 442-445).

Figure 2. (Left), importance of geomorphological processes as a function of average annual temperature and total annual rainfall, according to Peltier (1950). (Right), current morpho-climatic regions, from the average annual temperature, total annual rainfall and number of wet months above $50 \mathrm{~mm}$, according to Chorley et al. (1984); cited by Gutiérrez (2009, pp. 442-445).
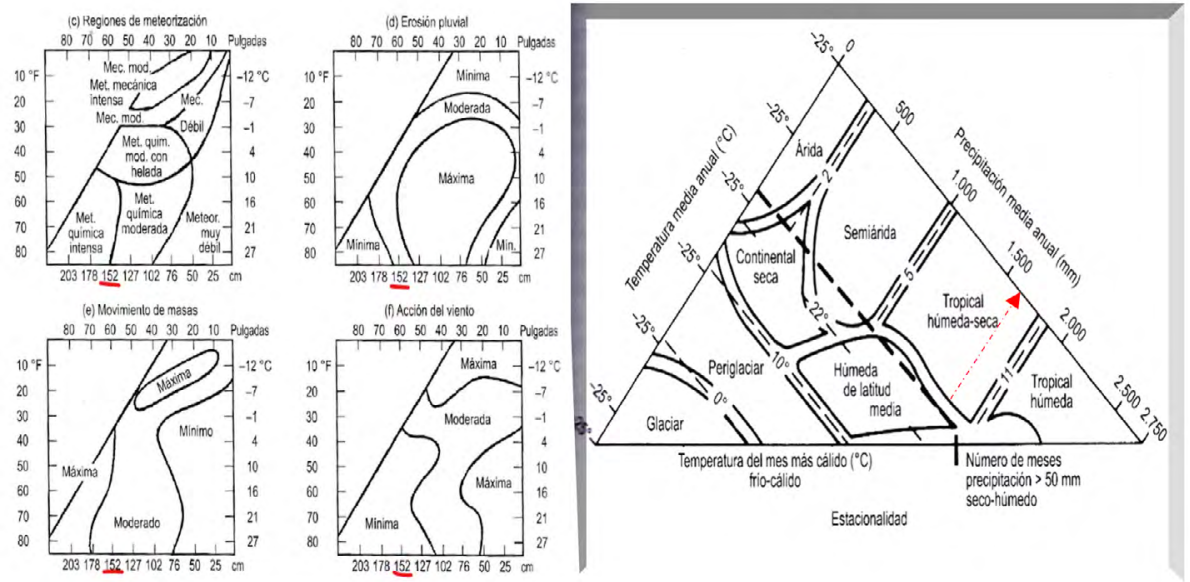

Con los datos de campo se delimita el geosistema y se elabora el mapa geomorfológico a escala 1:4 000. Se cuantifica el espacio y se identifican ocho (8), geofacies colindantes con la planicie costera, sobre la cual yace el manto de arenas (Figura 3 ).

- $\quad$ Contactos litológicos. Ígneo/Ignimbritos (0,82 ha), que conforman la punta expuesta. Sedimentario/ Arenas (2,1 ha), que constituyen el supralitoral al sur-este del polígono.

- $\quad$ Formas de origen marino. Playas de arenas: Norte 5,1 ha., sur 8,1 ha. Playa rocosa: 3,8 ha. Manglar: 21,7 ha. 
- $\quad$ Formas de origen eólico. Dunas litorales con vegetación: Se forman sobre el supralitoral de la playa de arenas $\mathrm{N}$. Abarcan un polígono de 1,1 ha. Se extienden en dirección de $\mathrm{N}$ a S por 435,5 m., presentando acumulaciones embrionarias de $0,80 \mathrm{~cm}$. hasta alcanzar los $7 \mathrm{~m}$. de altura. Presentan ripples bien definidos con longitudes de onda de \pm $8 \mathrm{~cm}$., y entre su base de barlovento y sotavento alcanzan $\pm 25 \mathrm{~m}$ de ancho. Dunas nebkhas: Se encuentran en un polígono de 11, 7 ha., donde convergen con los mangles de la planicie fluviomarina. Como dunas de sombra, se encuentran fitoestabilizadas, y alcanzan alturas de $\pm 15 \mathrm{msnm}$. Hoyo de deflación: Se encuentra ubicado entre las dunas litorales y nebkhas $\pm 40 \mathrm{~m}$. de ancho; delimita un polígono de 1,5 ha. Manto de arenas: Revestido con una película de arena de \pm 7 cm., circunscribe un área de 96,2 ha.

El tamaño del clasto es una de las propiedades física de mayor relevancia que se debe conocer para establecer las condiciones dinámicas que determinan el depósito de los sedimentos (Pérez y Márquez, 2017, p.21). Atendiendo los parámetros preestablecidos en la escala de Wentwoth, para clasificar por tamaño las partículas de los sedimentos (Kenitiro, 1973, p.36; Robinson, 1990, p.315; Pérez y Márquez, 2017, p.22), se realiza el análisis granulométrico por tamizado, el cual permite catalogar las muestras en tres categorías: Material selecto (constituido por conchas y materia vegetal retenidos por el tamiz $\mathrm{N}^{\mathrm{o}} 35$ ), arenas (clastos retenidos del tamiz $\mathrm{N}^{\mathrm{o}} 60$ al 230), limo y arcilla (todas las partículas de fondo que traspasaron el tamiz 230) (Tabla 2).

Un alto contenido de materia orgánica (MO), en el suelo, facilita una alta reserva de nutrientes y su capacidad para degradar contaminantes, garantizando su nivel de productividad. Sobre el suelo, la MO se caracteriza por ser un gran reservorio de carbono $(\mathrm{C})$, de alta residencia, y como sus concentraciones varían en el perfil del suelo, se recomienda realizar el análisis por horizonte (Carreira, 2011, p.1).

Después de 30 minutos de aplicado el procedimiento (sobre la muestra de $1 \mathrm{~g}$. de la planicie costera), la muestra exhibe un color naranja brillante, el cual sugiere, según tabla de bajo contenido de materia orgánica valores $<0.13 \%$ de trazas de materia orgánica. Veinticuatro horas después, 
Figura 3. Unidades geomorfológicas de la zona de pesquisa, a de la a escala 1:4000.

Figure 3. Geomorphological units of the research area, at a scale of 1: 4000

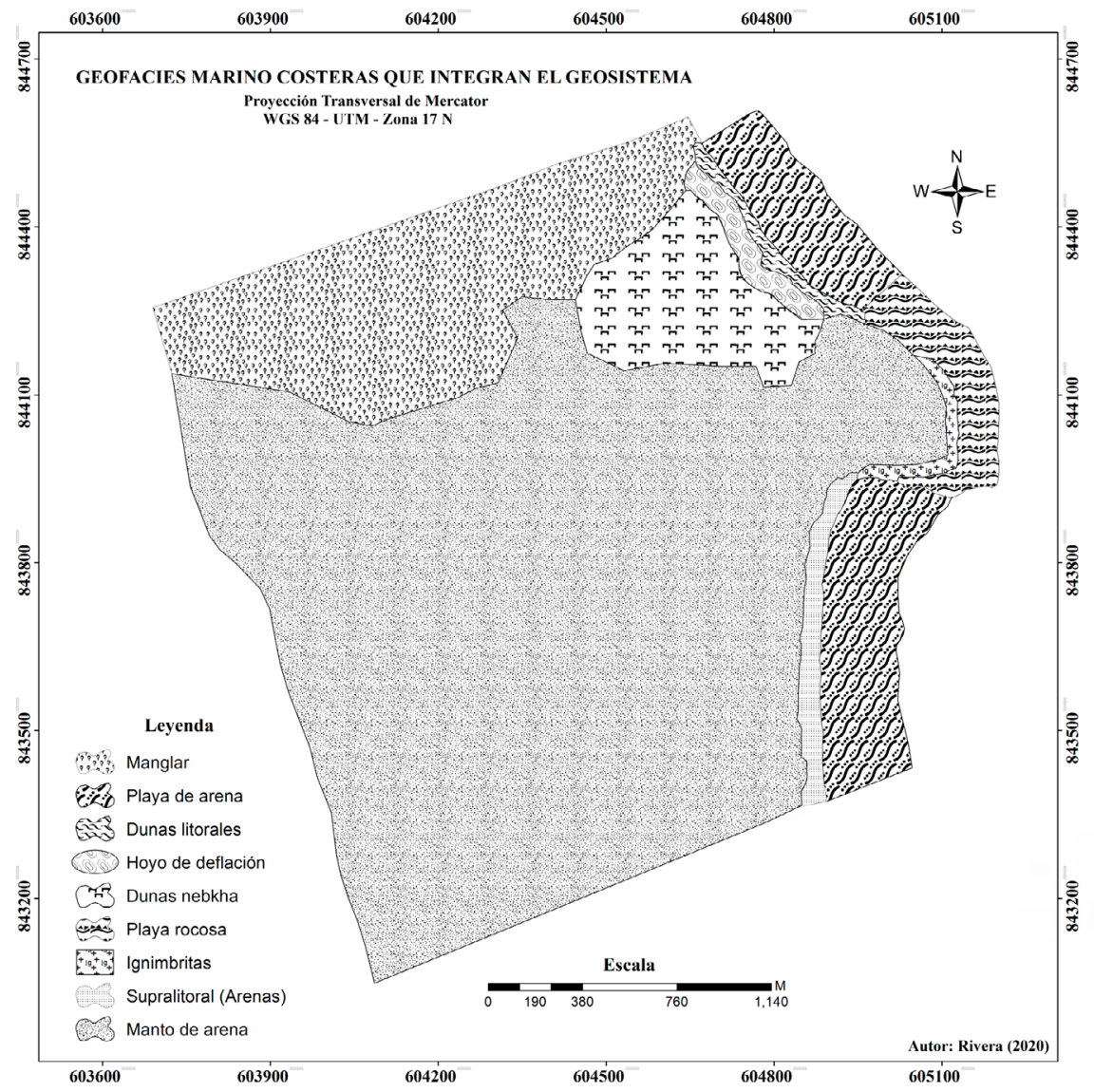

sobre el papel filtro, la muestra seca al aire aún conserva la totalidad de su peso inicial $(1 \mathrm{~g})$. Aplicado el método de reducción de materia orgánica, se comprueba que las partículas de arena que recubre la planicie costera no son producto de la meteorización del suelo In situ. 
Depósitos eólicos del trópico húmedo: caso de la franja marino-costera del este de la Península de Azuero, Panamá

Tabla 2. Clasificación de las partículas y distribución porcentual de la masa por tamaño.

Autor. Rivera (2019).

Table 2. Classification of particles and percentage distribution of mass by size.

Author. Rivera (2019).

\begin{tabular}{|c|c|c|c|c|c|c|}
\hline Clasificación & $\begin{array}{c}\text { Material } \\
\text { selecto }\end{array}$ & $\begin{array}{l}\text { Arena } \\
\text { media }\end{array}$ & Arena fina & $\begin{array}{l}\text { Arena muy } \\
\text { fina }\end{array}$ & $\begin{array}{l}\text { Limo y } \\
\text { arcilla }\end{array}$ & \multirow{3}{*}{$\begin{array}{c}\text { Total } \\
\text { Gramos }\end{array}$} \\
\hline $\mathrm{N}^{\mathrm{o}}$ Tamiz/ & $\begin{array}{c}>35 \\
\text { (Retenido) }\end{array}$ & $\begin{array}{c}60 \\
\text { (Retenido) }\end{array}$ & $\begin{array}{c}120 \\
\text { (Retenido) }\end{array}$ & $\begin{array}{c}230 \\
\text { (Retenido) }\end{array}$ & $\begin{array}{c}<230 \\
\text { (Fondo) }\end{array}$ & \\
\hline Unidad (mm) & $(0,50 \mathrm{~mm})$ & $(0,25 \mathrm{~mm})$ & $(0,125 \mathrm{~mm})$ & $(0,0625 \mathrm{~mm})$ & $(0,0625 \mathrm{~mm})$ & \\
\hline $\begin{array}{c}\text { Dunas } \\
\text { litorales }\end{array}$ & & $\begin{array}{l}30 \mathrm{~g} / \\
6,62 \% \\
\end{array}$ & $\begin{array}{l}382 \mathrm{~g} / \\
84,33 \% \\
\end{array}$ & $\begin{array}{l}38 \mathrm{~g} / \\
8,39 \% \\
\end{array}$ & $\begin{array}{c}3 \mathrm{~g} / \\
0,66 \% \\
\end{array}$ & $\begin{array}{c}453 \mathrm{~g} / \\
100 \% \\
\end{array}$ \\
\hline $\begin{array}{c}\text { Dunas } \\
\text { nebkhas }\end{array}$ & & $\begin{array}{c}8 \mathrm{~g} / \\
1,77 \%\end{array}$ & $\begin{array}{l}414 \mathrm{~g} / \\
91,39 \%\end{array}$ & $\begin{array}{l}28 \mathrm{~g} / \\
6,18 \%\end{array}$ & $\begin{array}{c}3 \mathrm{~g} / \\
0,66 \%\end{array}$ & $\begin{array}{c}453 \mathrm{~g} / \\
100 \%\end{array}$ \\
\hline $\begin{array}{l}\text { Manto de } \\
\text { arenas }\end{array}$ & $\begin{array}{l}16 \mathrm{~g} / \\
3,53 \%\end{array}$ & $\begin{array}{c}48 \mathrm{~g} / \\
10,60 \%\end{array}$ & $\begin{array}{l}342 \mathrm{~g} / \\
75,50 \%\end{array}$ & $\begin{array}{l}26 \mathrm{~g} / \\
5,74 \%\end{array}$ & $\begin{array}{l}21 \mathrm{~g} / \\
4,63 \%\end{array}$ & $\begin{array}{c}453 \mathrm{~g} / \\
100 \%\end{array}$ \\
\hline $\begin{array}{l}\text { Playa de arenas } \\
\text { (Mesolitoral N) }\end{array}$ & $\begin{array}{c}76 \mathrm{~g} / \\
16,78 \%\end{array}$ & $\begin{array}{l}18 \mathrm{~g} / \\
3,97 \%\end{array}$ & $\begin{array}{l}296 \mathrm{~g} / \\
65,34 \%\end{array}$ & $\begin{array}{c}62 \mathrm{~g} / \\
13,69 \%\end{array}$ & $\begin{array}{c}\mathrm{g} / \\
0,22 \%\end{array}$ & $\begin{array}{c}453 \mathrm{~g} / \\
100 \%\end{array}$ \\
\hline
\end{tabular}

Durante el transporte de los sedimentos por el viento, el proceso de abrasión continúa desgastando los clastos de arenas, tornárnoslo redondos y esféricos; además, como resultando del transporte, cuando casi todas las partículas tienen el mismo tamaño se concluye que los sedimentos depositados se encuentran bien seleccionados (Wicander y Monroe, 2009, pp.342-345).

Por tanto, se aceptable calcular la redondez y esfericidad de una partícula comparándola con un grupo de modelos de referencia, donde, valores numéricos entre 0 a 1 determinan la proximidad de los clastos a un nivel de esfericidad y redondez altos (Robinson, 1990, p.350).

En consecuencia, auxiliados con la tabla de comparación visual (Figura 4), el análisis morfoscópico por muestreo aleatorio simple y comparado entre los clastos de cuarzo demuestra que los del mesolitoral se exhiben subangulosos - subprismático y subredondeados - subesferoidal (Coeficientes de redondez y esfericidad entre 0,5 y 0,7$)$, mientras que los de la planicie 
costera son subredondeados - subesferoidal y redondeados - esferoidal (Coeficientes de redondez y esfericidad entre 0,7 y 0,9 ), (Figura 5).

Figura 4. Tabla de comparación visual de redondez y esfericidad de arena según Krumbein y Sloss (1963). (Kenitiro, 1973, p. 113).

Figure 4. Visual comparison table of roundness and sphericity of sand, according to Krumbein and Sloss (1963). (Kenitiro, 1973, p. 113).

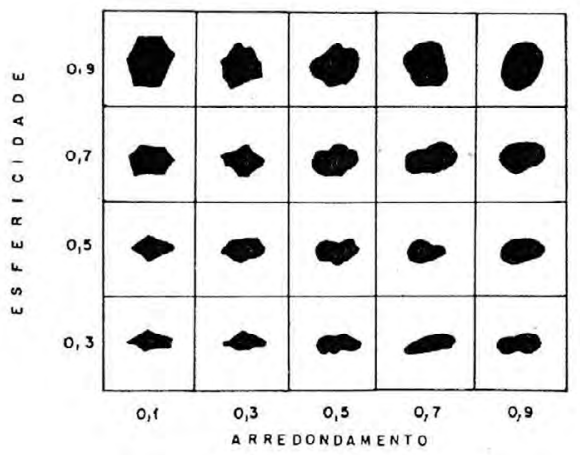

Figura 5. (Izquierda), clastos de cuarzo del mesolitoral (Playa de arenas N). (Derecha), clastos de cuarzo sobre la planicie costera (Manto de arenas).

Autor: Rivera (2020).

Figure 5. (Left), mesolittoral quartz clasts (Sandy beach N). (Right), quartz clasts on the coastal plain (Mantle of sands).

Author: Rivera (2020).
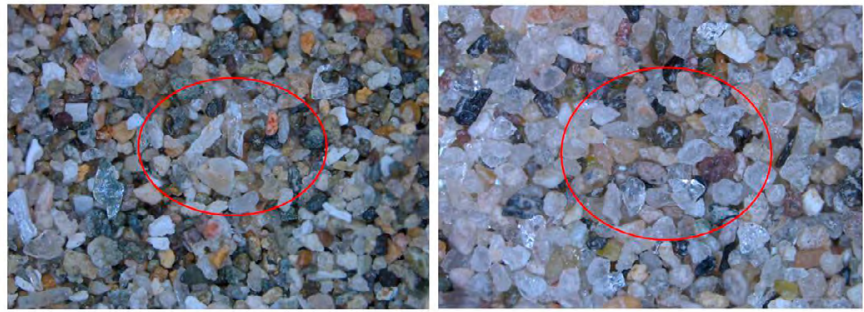
Puesto que el material selecto estaba constituido por fragmentos de materia vegetal sobre el manto de arenas, y conchas sobre la playa de arenas, de los resultados de la tabla 2, se eligieron las arenas del mesolitoral como variable independiente para evidenciar la correlación granulométrica entre éstas y el origen de los depósitos eólicos que componen el ambiente sedimentario; y como variable dependiente, se utilizó el total de la masa retenida de arena media, arena fina, arena muy fina y de fondo, la de limo y arcilla por geofacie (Figuras 6 y 7).

También, se utilizan las dunas litorales como variable independiente para correlacionar con las dunas nebkhas y el manto de arenas (Figura 8); en todos los casos, se determina una correlación lineal positiva de coeficiente perfecto $>0,957$.

Figura 6. Variable independiente playa de arenas. (Izquierda), correlación granulométrica con las dunas litorales. (Derecha), correlación granulométrica con las dunas nebkhas.

Autor: Rivera (2020).

Figure 6. Independent variable sandy beach. (Left), granulometric correlation with the coastal dunes. (Right), granulometric correlation with the Nebkhas dunes.

Author: Rivera (2020).
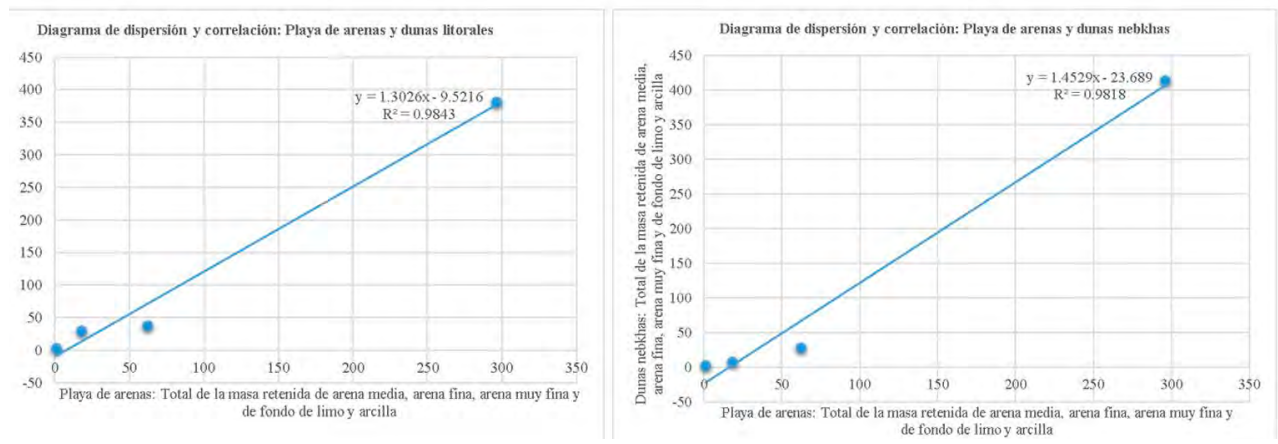
Figura 7. Variable independiente playa de arenas. Correlación granulométrica con el manto de arenas.

Autor: Rivera (2020).

Figure 7. Variable independent sandy beach. Granulometric correlation with the sands mantle.

Author: Rivera (2020)

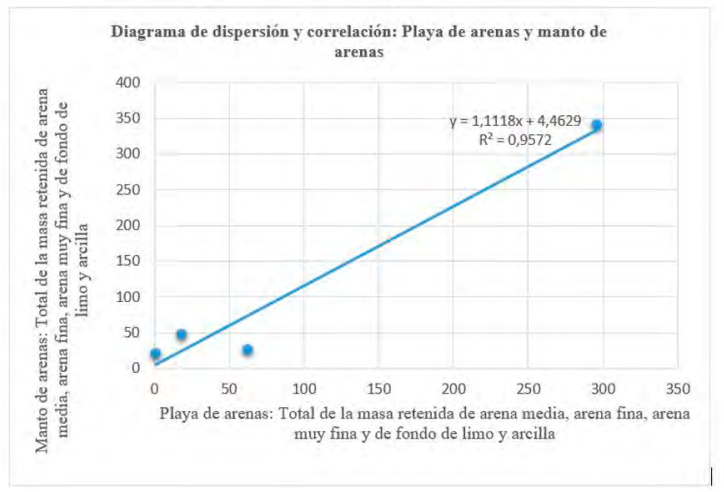


Depósitos eólicos del trópico húmedo: caso de la franja marino-costera del este de la Península de Azuero, Panamá

Figura 8. Variable independiente dunas litorales. (Superior), correlación granulométrica con las dunas nebkhas. (Inferior), correlación granulométrica con el manto de arenas.

Autor: Rivera (2020).

Figure 8. Independent variable coastal dunes. (Superior), granulometric correlation with the Nebkhas dunes. (Bottom), granulometric correlation with the sands mantle.

Author: Rivera (2020).
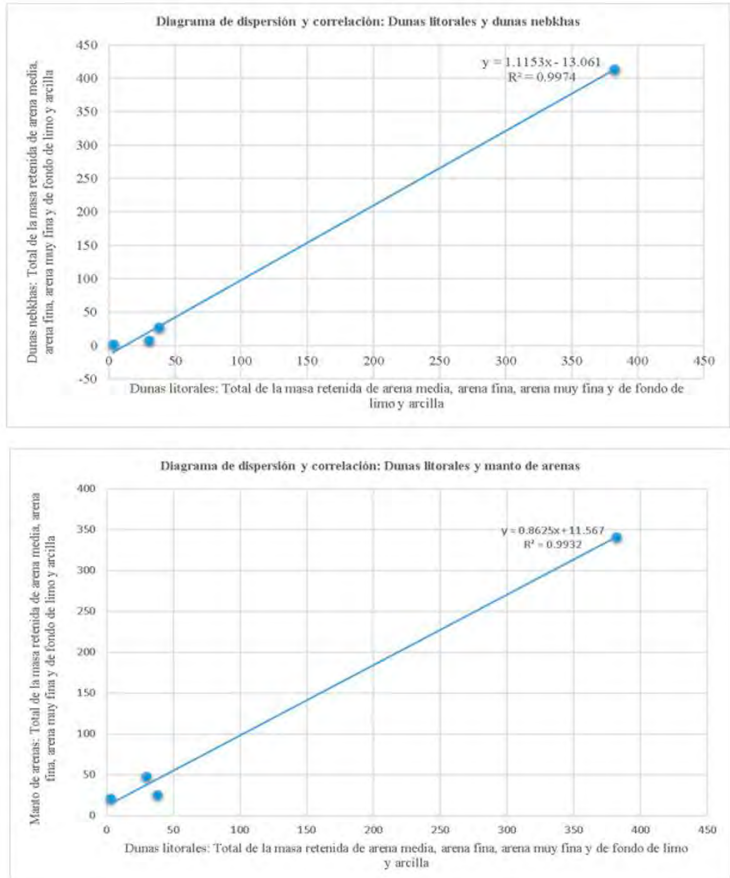


\section{Discusión de los resultados}

La geomorfología, el tipo de roca, las características climáticas, así como la energía que genera el transporte y depósito de los sedimentos, originan diferentes texturas que deben ser evaluadas al realizar las interpretaciones del ambiente sedimentario (Kenitiro, 1973, p.182; Pérez y Márquez, 2017, p.56). Se aplica el concepto de textura del relieve cuando se procura resaltar o destacar el nivel de cercanía o distancia que presentan las unidades morfológicas que integran el paisaje, generalmente a escala local.

Por ejemplo, para Patton, Alexander y Kramer (1983, p.266), la textura de una forma del relieve se refiere al espaciamiento de los elementos que integran el espacio, donde, se consideran de textura fina o estrechamente vinculadas cuando se encuentran poco separadas. Esta caracterización resulta frecuente en los ambientes sedimentarios, los cuales, se definen como aquel espacio geográfico donde los sedimentos son depositados (Wicander y Monroe, 2009, p.345-349; Tarbuck y Lutgens, 2013, p.215).

Uno de estos espacios, son los mantos de arenas o mantos eólicos, los cuales son acumulaciones de arena con morfologías planas o poco onduladas que recubren las irregularidades del substrato con espesores que oscilan entre los pocos centímetros hasta sobrepasar el metro; sus arenas suelen tener un tamaño de muy fino, fino, medio y grueso (Bernat y Pérez, 2005, p.25).

En consecuencia, el plano geomorfológico (Figura 3), demuestra el espacio requerido y el reconocimiento de la forma adquirida por cada geofacie en respuesta a los flujos de energía dentro del geosistema, donde, las geoformas resultantes demuestran los que es una textura fina del relieve en un ambiente deposicional de transición marino costero (Figura 9).

Este ambiente sedimentario es sin duda producto del factor eólico, que agudiza sus efectos durante el periodo seco del clima húmedo tropical; propiciado también, por la previa existencia de sedimentos que pueden ser transportados (arenas), acarreados y depositados en la franja sublitoral y mesolitoral por procesos fluviales (existencia de estuarios), y marinos (corrientes costaneras o deriva litoral).

Éste ambiente sedimentario marino costero de clima húmedo se caracteriza por la presencia, acumulación y dispersión de sus arenas; las 
cuales, están compuestas predominantemente por partículas de cuarzo, que adquirieron formas bien redondeadas y casi esféricas.

Además, las geoformas resultantes están compuestas de arena fina, por más del 75,5\% del total de la muestra; lo cual permite clasificarlas como geoformas constituidas por arenas bien seleccionadas, evidencia del transporte eólico. La erosión eólica ocurre por deflación, entendida como el proceso que conjuga la movilización, el transporte y la depositación del clasto cuando la fuerza del viento ha disminuido su energía, y, por tanto, su capacidad de mover los clastos.

$\mathrm{Al}$ respecto, las arenas entre $0,04 \mathrm{~mm}$ a $0,40 \mathrm{~mm}$ son la que se movilizan con mayor facilidad (Gutiérrez, 2009 pp.356-358), para Blyth y de Freitas (1989, p.79), vientos de $4 \mathrm{~m} / \mathrm{s}$ son suficientes para desplazar arenas $<0,35 \mathrm{~mm}$; sin embargo, los clastos con diámetros $>1 \mathrm{~mm}$ necesitan ráfagas de vientos de $5 \mathrm{~m} / \mathrm{s}$ (Robinson, 1990, p. 501).

Figura 9. Ambiente deposicional marino costero. (1), playa de arenas del litoral N, con ripples expuestos durante el periodo seco; (2), cara de barlovento de la duna litoral con formación de ripples por reptación;

(3), dunas litorales con vegetación; (4), cota de la duna litoral con microforma por reptación; (5), base erosiva de sotavento de la duna litoral hacia el hoyo de deflación; (6), fondo del hoyo de deflación; (7), mesoformas, lóbulo deposicional por saltación, dunas nebkhas; (8), cota de las dunas nebkhas; (9), colecta de arenas sobre la planicie costera a $\pm 1 \mathrm{~km}$ de la playa de arenas; (10), vista de la planicie costera donde se genera el manto de arenas.

Figure 9. Coastal marine depositional environment. (1), sandy beach of the $\mathrm{N}$ coast, with ripples exposed during the dry period; (2), windward side of the coastal dune with formation of ripples by creep; (3), coastal dunes with vegetation; (4), elevation of the coastal dune with creeping microform; (5), erosive leeward base of the coastal dune towards the deflation pit; (6), deflation pit bottom; (7), mesoforms, depositional lobe by jumping, nebkhas dunes; (8), elevation of the Nebkhas dunes; (9), collection of sands on the coastal plain $\pm 1 \mathrm{~km}$ from the sandy beach;

(10), view of the coastal plain where the sand cover is generated 
Jaime A. Rivera-Solis Aeolian deposits of the humid tropics: case of the marine-coastal strip of the eastern part of the Azuero Peninsula, Panama

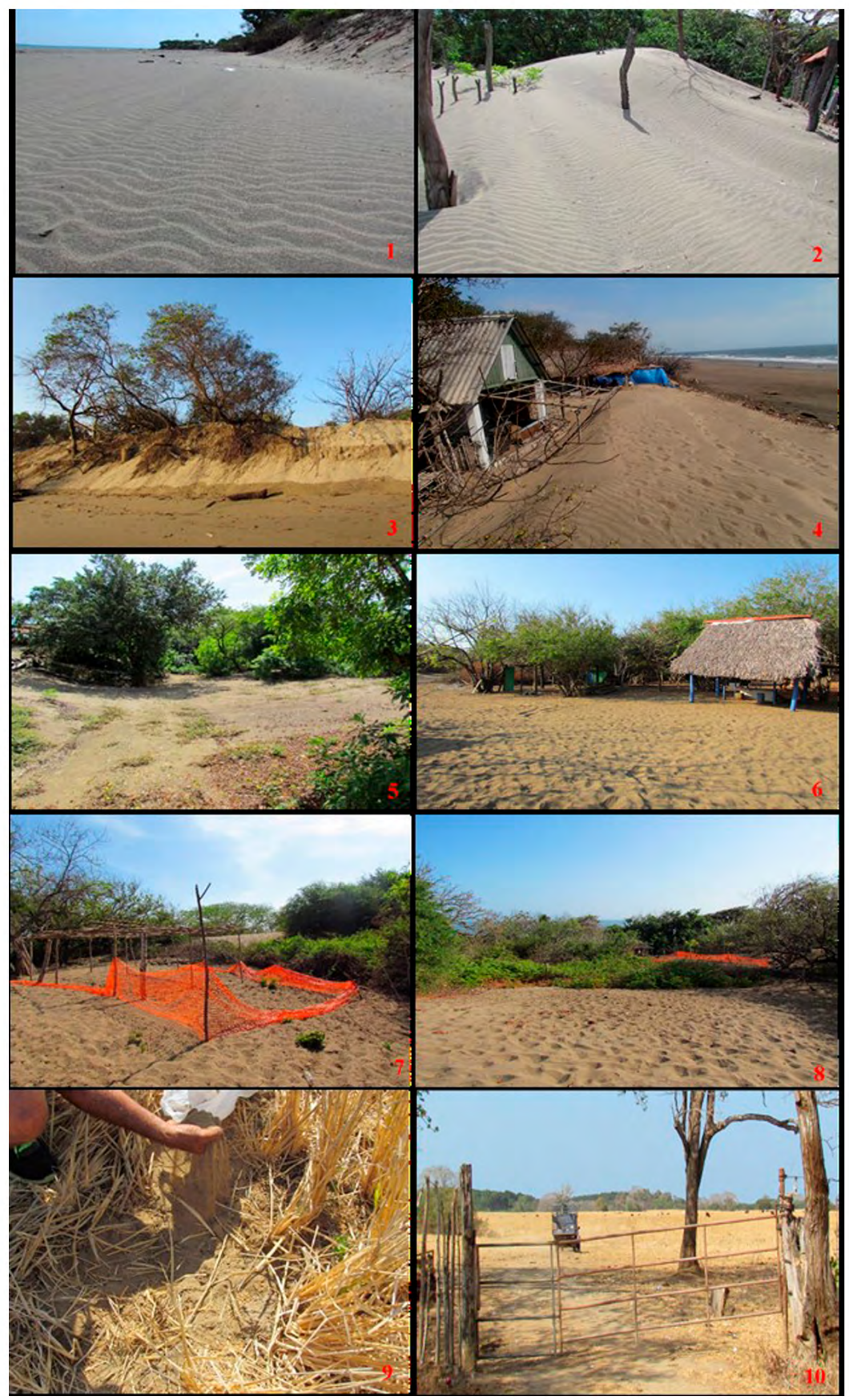

120 Revista Geográfica de América Central. No 66(1)

ISSN 1011-484X • e-ISSN 2215-2563 / Enero-junio 2021

Doi: https://dx.doi.org/10.15359/rgac.66-1.4 
Estas teorías permiten discutir la relación entre la velocidad del viento y el tamaño de los clastos seleccionados y depositados por geoforma identificada. Correlacionando todas las variables investigadas se comprueba que en un clima tropical húmedo estacional se desarrollan ambientes sedimentarios de transición donde las geofacies evolucionan en secuencia a partir del mismo material de origen, la arena, y como factor morfogenético, el viento.

Por tanto, los resultados permiten elaborar los siguientes postulados:

(a) Como región morfoclimatica, en los climas tropicales húmedos ( $>1500 \mathrm{~mm}$ de precipitación total anual), que presenten periodos estacionales y vientos constantes $>5 \mathrm{~m} / \mathrm{s}$, es posible el desarrollo de ambientes sedimentarios; vinculados estrictamente a la existencia de geoformas de acumulación o de erosion, cuyo material parental residual serán arenas.

(b) Derivados de la fuerza morfoenetica del viento, en los ambientes sedimentarios de las zonas marino costeras del trópico húmedo es posible encontrar geofacies de erosion (Hoyos de deflación), y geofacies de acumulación como dunas litorales, dunas nebkhas y mantos de arena.

(c) En la zona de estudio, el viento no solo modela las geofacies. Sino que, es posible comprobar sus efectos sobre las partículas constituyentes de cada geotopo. Así, a través del análisis morfoscópico (por muestreo aleatorio simple y comparado entre los clastos de cuarzo), se demuestra que los clastos del mesolitoral se exhiben subangulosos - subprismático y subredondeados - subesferoidal (coeficientes de redondez y esfericidad entre 0,5 y 0,7$)$, mientras que los de la planicie costera (Manto de arenas), son subredondeados - subesferoidal y redondeados - esferoidal (coeficientes de redondez y esfericidad entre 0,7 y 0,9$)$. Por tanto, se infiere que los clastos con coeficientes $>0,7$ son fácilmente movilizados (por rodadura, saltación o suspensión), y, en consecuencia, disminuyen sus ángulos y se tornan más redondeados.

(d) La génesis de los clastos de arena sobre las geoformas expuestas queda demostrada al utilizando como variable independiente los clastos de la Playa de Arenas N. Por correlación granulométrica y, según el coeficiente de correlación de Pearson; existe una correlación lineal 
positiva y de coeficiente perfecto de $\rho \pm 0,98$ con las Dunas Litorales, de $\rho \pm 0,98$ con las Dunas Nebkhas, y de $\rho \pm 0,96$ con el Manto de Arenas. También, utilizando como variable independiente los clastos de las Dunas Litorales, la correlación granulométrica demuestra, una correlación lineal positiva y de coeficiente perfecto de $\rho \pm 1$ con las Dunas Nebkhas, y de $\rho \pm 0,99$ con el Manto de Arenas.

(e) En atención a las recomendaciones de Blyth y De Freitas (1989), Simons (1990) y Gutiérrez (2008), aunado a los resultados de la tabla 2; se comprueba que el viento constituye en los climas estacionales húmedos un importante agente morfogenético que cuenta con la capacidad de crear ambientes sedimentarios. En la zona de estudio, durante el periodo seco los vientos que viajan a velocidades promedios desde 3 a $>5 \mathrm{~m} / \mathrm{s}$, cuentan con la energía suficiente para movilizar clastos de arenas con diámetros de $0,25 \mathrm{~mm}, 0,125 \mathrm{~mm}$ y $0,0625 \mathrm{~mm}$; desde el litoral hasta un (1) $\mathrm{km}$ de distancia dentro del continente, formando mantos de arenas sobre la planicie costera.

\section{Conclusiones}

La Geomorfología Ambiental propicia el análisis geosistémico del espacio natural, ponderando los procesos morfogenéticos y sus efectos sobre las unidades del paisaje (geotopos y geofacies); justo con el análisis de sus resultados, el geógrafo puede elaborar las propuestas de ordenación ambiental.

La zona de estudio expone una textura fina del relieve en un ambiente sedimentario de transición, constituido por dunas litorales, hoyo de deflación, dunas nebkhas, y finalmente mantos de arenas. La existencia de éstos depósitos eólicos en climas tropicales estacionales con precipitación total anual $>1500 \mathrm{~mm}$, obliga a documentar los efectos morfogenéticos del viento durante el periodo seco.

Si bien los efectos morfodinámicos del viento son considerados como insignificantes en los climas tropicales húmedos, sin embargo, durante el periodo seco, merece especial atención documentar la existencia de depósitos eólicos, puesto que, el transporte de arenas hacia adentro del continente estaría intensificando la acidificación de los suelos agrícolas de las planicies costeras; como queda demostrado por la disminución del pH 
de 5.6 durante el periodo lluvioso, a 4.2 durante el periodo seco, cuando se encuentra revestida por arena.

Al presentar las planicies costeras arenas finas bien seleccionadas, que representan más del $75 \%$ del total de las muestras y donde predominan los clastos de cuarzo, con valores $<0.13 \%$ de trazas de materia orgánica sobre el primer horizonte del suelo, y coeficientes de redondez y esfericidad entre 0,7 y 0,9 ; fundamenta el hecho de que, las arenas acumuladas durante el periodo seco sobre la planicie costera, son producto del proceso de deflación y no meteorizadas en In situ.

\section{Referencias}

Ayoade, J. (2011). Introdução à climatologia para os trópicos. (15 a ed). Rio de Janeiro, Brasil: Editora Bertrand LTDA.

Bernat, M. y Pérez, A. (2005). Campos de dunas y mantos e ólicos de Tierra de Pinares: Sureste de la cuenca del Duero. España. Boletín Geológico y Minero, 116 (1), pp. 23-38. Recuperado de: https://www. researchgate.net/publication/259226985_Campos_de_dunas_y_ mantos_eolicos_de_Tierra_de_Pinares_Sureste_de_la_cuenca_del_ Duero_Espana

Bertrand, G. y Bertrand, C. (2007). Uma Geografia transversal e de travessias: O meio ambiente através dos territórios e das temporalidades. Brasil: Editora Massoni.

Blyth, F. y de Freitas, M. (1989). Geología para ingenieros. México: Compañía editorial continental, S.A.

Carreira, D. (2011). Cuantificación de la Materia Orgánica del suelo. Método de Walkley \& Black. Trabajo presentado en la Jornada de Actualización: Gestión de la calidad en los laboratorios de análisis de suelos agropecuarios. SAMLA- PROINSA. Rosario, Argentina. Recuperado de: https://inta.gob.ar/sites/default/files/script-tmp-anlisis_de_c_y_n.pdf

Craig, J. R., Vaughan, D.J. y Skinner, B.J. (2012). Recursos de la Tierra y el medio ambiente. ( $4^{\mathrm{a}}$ ed). Madrid, España: Pearson Educación, S.A.

Díaz, R. (2011). Desarrollo sustentable: Una oportunidad para la vida. (2 ed). México D.F: Editora McGraw-Hill. 
Eyherabide, M., Saínz, H., Barbieri, P. y Echeverría, H. (2014). Comparación de métodos para determinar carbono orgánico en suelo. Ciencia del Suelo (Argentina) 32 (1), pp. 13-19. Recuperado de: https:// www.researchgate.net/publication/276917420_COMPARACION_ DE_METODOS_PARA_DETERMINAR_CARBONO_ORGANICO_EN_SUELO

Gutiérrez, M. (2009). Geomorfología. Madrid, España: Pearson Educación. Instituto Geográfico Nacional "Tommy Guardia". (2016). Atlas Nacional de la República de Panamá. ( $5^{\text {a }}$ ed). Colombia: Impresiones Carpal.

Kenitiro, S. (1973). Introdução à sedimentolologia. São Paulo, Brasil: Editora Edgard Blucher Ltda.

Mateo, J. (2012). La dimensión espacial del desarrollo sostenible: Una visión desde América Latina. La Habana, Cuba: Editorial UH.

Ministerio de Vivienda y Ordenamiento Territorial. (2006). Ley 6; por la cual se reglamenta el Ordenamiento Territorial para el desarrollo urbano y se dicta otras disposiciones. Recuperado de: https://docs. panama.justia.com/federales/leyes/6-de-2006-feb-3-2006.pdf

Ministerio de Vivienda y Ordenamiento Territorial. (2009). Resolución No 4; por la cual se establece el procedimiento y los requisitos para la tramitación de solicitudes relacionadas con el ordenamiento territorial para el desarrollo urbano. Recuperado de: https://www.gacetaoficial.gob.pa/pdfTemp/26221/16057.pdf

Patton, C., Alexander, C. y Kramer, F. (1983). Curso de Geografía Física. España: Ediciones Vicens-Vives.

Peel, M.C., Finlayson, B.L. y McMahon, T.A. (2007). Updated world map of the Köppen-Geiger climate classification. Recuperado de: https:// www.hydrol-earth-syst-sci.net/11/1633/2007/hess-11-1633-2007. pdf

Pérez, A. y Márquez, A. (2017). Sedimentología. Universidad Autónoma Metropolitana, Unidad Iztapalapa (División de Ciencias Biológicas $y$ de la Salud). México. Impreso México D.F.

Rivera, J. (2016). Avaliação geoecológica aplicada à ordenação ambiental da paisagem marinho-costeira: caso da bacia hidrográfica do Rio Purio Província dos Santos - Panamá (Tesis doctoral). Instituto de Geociências e Ciências Exatas, Departamento de Geografia, Universidade Estadual Paulista. Brasil. (p.198). 
Robinson, E. (1990). Geología física básica. México: Editorial limusa, S.A.

Sánchez, J. (2003). Geomorfologia: Ambiente e Planejamento. ( $7^{\mathrm{a}}$ ed.). São Paulo, Brasil: Editora Contexto.

Schober, P. Boer, C y Schwarte, L. (2018). Correlation coefficients: Appropriate use and interpretation. Journal Anesthesia \& Analgesia (United States of America), 126 (5), pp. 1763-1768. Recuperado de: https://journals.lww.com/anesthesia-analgesia/fulltext/2018/05000/ correlation_coefficients_appropriate_use_and.50.aspx

Tarbuck, E. y Lutgens, F., 2013, p. 251. Ciencias de La Tierra. Una Introducción a la Geología Física. (10ª ed). Madrid, España: Pearson Educación.

Teixeira, A. y Dos Santos, M. (2015). Geomorfología Ambiental. ( $\left.7^{\mathrm{a}} \mathrm{ed}\right)$. Rio de Janeiro, Brasil: Bertrand Brasil.

Troppmair, H. (2012). Biogeografia e meio ambiente. ( $9^{\mathrm{a}}$ ed). Rio de Janeiro, Brasil: Technical books editora.

Verstapen, H.T. y Zuidam, R.A. (1975). ITC System of geomorphological survey. Netherlands: Manuel ITC Textbook. Vol. VII, Chapter VII.3. Wicander, R. y Monroe, J. (2009). Fundamentos de Geología. São Paulo, Brasil: Cengage Learning Edições Ltda. 
